\title{
Bees may enjoy coke
}

Evolutionary mechanisms of recreational drug use have puzzled researchers for years. Many drugs, among them cocaine, function as natural insecticides that protect the plants in which they are produced. This creates a paradox: why would a substance that evolved to deter insects be attractive and rewarding to mammals? One proposed solution is that the brains of insects and mammals simply work differently, meaning that certain drugs trigger reward mechanisms in mammals but not in insects. Now, scientists from the University of Illinois (Urbana) and from the Australian National University (Canberra) refute this theory by showing that honeybees, like humans, seem to derive pleasure from ingesting cocaine.

The researchers, led by Gene Robinson of the University of Illinois, assessed bees' perception of reward by evaluating their performance in a 'waggle dance' (J. Exp. Biol. 212, 163-168; 2009). Bees use this sophisticated dance to communicate to their nestmates the precise location and quality of food resources ('rewards') they have discovered. Robinson and colleagues

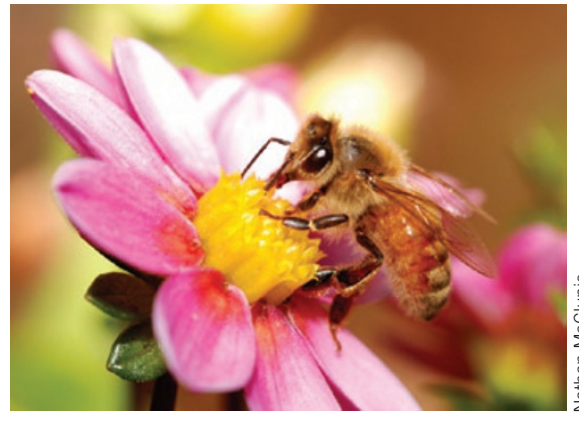

bees. The effect was dose-dependent, and bees that were chronically treated with cocaine suffered withdrawal symptoms after treatment ceased.

To counter suggestions that cocaine merely triggered a motor response and not a reward response, the researchers showed that aside from changes to the dance, bees' motor function was normal. Furthermore, cocaine-treated bees that were separated from the hive did not spontaneously break out into dance; bees performed administered low doses of cocaine to honeybees as they fed at sucrose and pollen feeders. The researchers then recorded bees' movements upon returning to the hive. Compared with untreated bees, bees that were treated with cocaine danced more often and more rapidly after discovery of either sucrose or pollen. In a natural setting, a bee would intensify its dance this way to report a food resource of a higher value, meaning that cocainetreated bees perceived a greater reward than they actually discovered. This suggests that contrary to previous assumptions, cocaine triggers reward mechanisms in these movements only in the appropriate social context of returning to the hive after foraging and communicated clear, though exaggerated, information. "It's not like they're gyrating wildly on the dance floor out of control," explained Robinson.

Cocaine kills herbivorous insects by disrupting motor control. Because the same neurochemical pathways that regulate motor control are involved in reward processing, Robinson and colleagues suggest that the rewarding properties of cocaine might be "side effects" of the drug's action as an insecticide.

Karen Marron

\section{BACTERIAL INFECTION MAY LIMTT SPREAD OF DENGUE FEVER}

Dengue fever is a viral febrile disease caused by flaviviruses carried by the Aedes aegypti mosquito vector. It affects tens of millions of people per year, primarily in tropical and African countries. The primary mode of prevention is through mosquito control, but typical mosquito control measures, such as using bed nets and draining areas of standing water, are less effective against $A$. aegypti mosquitoes because they are day-biters and thrive in urban areas.

Now, a group of researchers from University of Queensland (Brisbane, Australia) reports success with a new method of mosquito control: shortening mosquito lifespan via infection with the bacterium Wolbachia pipientis. Because the virus that causes dengue requires an incubation period of about 2 weeks in its mosquito vector before it can infect humans, only older mosquitoes pose a potential dengue threat. Shortening the lifespan of infected mosquitoes may thus be an effective means of reducing dengue transmission.

Wolbachia bacteria infect a range of arthropods. They require female hosts in order to survive and have developed certain strategies to increase the prevalence of females in their host populations. One such mechanism is cytoplasmic incompatibility, in which matings between uninfected females and infected males produce no offspring. These strategies enable Wolbachia sp. to spread rapidly in host populations. Paired with its ability to shorten lifespan, this characteristic makes W. pipientis a potentially valuable tool for controlling insect populations. Unfortunately, Wolbachia sp. do not naturally infect $A$. aegypti.

The University of Queensland researchers, led by Scott O'Neill, looked to circumvent this obstacle. They chose a strain of W. pipientis that normally infects Drosophila melanogaster fruit flies and causes them to die early. Initial attempts to infect $A$. aegypti with W. pipientis were unsuccessful, so $0^{\prime}$ Neill's group adapted the bacterium by culturing it with mosquito cells for 3 years. The adapted W. pipientis successfully infected female $A$. aegypti and reduced the mosquitoes' lifespan by half, from 60 days to around 30 days (Science 323, 141-144; 2009). W. pipientis infection was stable. O'Neill's team then screened larvae from infected $A$. aegypti females to evaluate the maternal transmission rate of W. pipientis and found that it was very high ( $>99 \%)$.

These results suggest that infection with $W$. pipientis could be an effective means of controlling $A$. aegypti populations and the transmission of dengue fever. Scientists caution, however, that field studies are needed to determine how well this approach might work under more natural conditions.

Monica Harrington 